\title{
Chlamydia trachomatis in gonococcal and postgonococcal urethritis
}

\author{
PERTTI TERHO \\ From the Department of Dermatology and Venereology and Department of Virology, \\ University of Turku, Finland
}

SUMMARY Chlamydia trachomatis was isolated from the urethra of $38(28.6 \%)$ out of 133 men with gonococcal urethritis (GU). During the follow up of 72 men postgonococcal urethritis (PGU) was diagnosed in $50(69.5 \%)$ patients. More than half (30 out of 50) of these patients with PGU were Chlamydia-positive. Out of 31 patients with Chlamydia 30 developed PGU whether or not procaine penicillin, spectinomycin, or gentamicin were used. These findings are discussed in relation to present recommendations for the treatment and follow up of patients with GU.

\section{Introduction}

In a great number of patients with gonococcal urethritis (GU) an urethral inflammatory reaction remains after the successful elimination of Neisseria gonorrhoeae (Vaughan-Jackson et al., 1977). This postgonococcal urethritis (PGU) is generally accepted as the equivalent of non-specific urethritis (NSU) contracted simultaneously with GU (Oriel et al., 1975) but causing symptoms or signs of urethritis later because of a longer incubation period (Oriel et al., 1972; Terho, 1978a).

Chlamydia trachomatis is believed to be the cause of NSU in about $50 \%$ of cases (Oriel, 1976). In addition, $C$. trachomatis is not sensitive to the agents most often used for the treatment of GU, such as penicillin (Johnson and Hobson, 1977) or ampicillin (Ridgway et al., 1976).

C. trachomatis is isolated in about one third of patients with GU (Richmond et al., 1972), this figure being higher when GU is followed by PGU and after treatment with antimicrobials which do not affect the growth of $C$. trachomatis, such as gentamicin (Oriel et al., 1975), kanamycin (VaughanJackson et al., 1977), or spectinomycin (Oriel et al., 1977).

In the present study the isolation rate of $C$. trachomatis is reported in 133 men with GU. These men were treated with procaine penicillin or an aminoglycoside (gentamicin or spectinomycin) and were subsequently examined for the development of PGU.

\footnotetext{
Address for reprints: Dr P. Terho, Department of Virology, University of Turku, Kiinamyllynkatu 10, SF-20520 Turku 52, Finland Received for publication 18 April 1978
}

\section{Materials and methods}

STUDY POPULATION

Heterosexual men were investigated following examination at either the Turku clinic for venereal diseases or the Department of Dermatology and Venereology, University of Turku, Finland, between February 1974 and February 1976.

Initially these 135 men had GU diagnosed by Gram-staining of the urethral exudate and by culture. The initial culture for $C$. trachomatis was grossly contaminated in two $(1.5 \%)$ of them, and they were thus excluded. The mean age of the remaining 133 patients with GU was $27 \cdot 4$ years.

SPECIMENS AND LABORATORY METHODS

At each visit all the men were examined for urethral inflammatory reaction by means of Gram-staining of smears of urethral exudate. Urethritis was diagnosed if 20 or more polymorphonuclear leucocytes were seen in more than two microscopic fields (magnification $\times 800)$ (Oriel et al., 1975). The urethral material from every patient was cultured for $N$. gonorrhoeae on a selective medium and for C. trachomatis using the irradiated McCoy cell culture technique (Terho, 1978b). The same examinations were performed at every visit.

\section{STUDY PLAN}

After GU was diagnosed the patients were treated either with 2.4 megaunits of procaine penicillin intramuscularly, or with $280 \mathrm{mg}$ gentamicin (Garamycin, Schering), after careful questioning about auditory or renal abnormalities, or with $2 \mathrm{~g}$ spectinomycin hydrochloride (Trobicin, Upjohn) intramuscularly. The choice of treatment was arbitrary. 
Patients were asked to refrain from sexual activity and to return for follow-up examination after one week. Special attention was then paid to the development of PGU and appropriate treatment was begun if needed. At this stage only the results of the gonococcal cultures were known. All cultures for Chlamydia were performed after the patient had been treated, usually many months later.

\section{STATISTICS}

Statistical analysis was made with Yates's $\chi^{2}$ test. Statistical significance was regarded as almost significant $(P<0.05)$, significant $(P<0.01)$, or highly significant $(\mathrm{P}<0.001)$.

\section{Results}

INITIAL ISOLATION OF C. TRACHOMATIS Initially, urethral specimens from 133 men with GU were cultured for $C$. trachomatis; of these 38 $(28.6 \%)$ were Chlamydia-positive.

FOLLOW UP AND DEVELOPMENT OF PGU Of the 133 men with GU $72(54 \cdot 1 \%)$ were followed up (Figure). Of the 61 men who defaulted 12 $(19.7 \%)$ had Chlamydia-positive cultures and 49 $(80 \cdot 3 \%)$ were Chlamydia-negative.

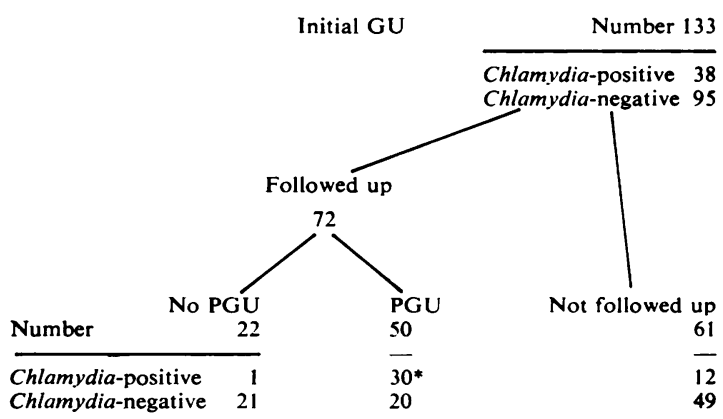

*Includes five patients with initially Chlamydia-negative cultures.

Figure Number of patients with gonococcal urethritis $(G U)$ and postgonococcal urethritis ( $P G U)$

Of the 72 men followed up $26(36 \cdot 1 \%)$ had Chlamydia-positive cultures initially. During follow up PGU was not diagnosed in $22(30.5 \%)$ patients, only one of these men without PGU having a positive urethral culture for $C$. trachomatis. However, PGU was diagnosed in $50(69.5 \%)$ of these 72 patients. Initially, 25 of them had Chlamydia-positive cultures, but during follow up cultures from five more men became Chlamydia-positive. Thus 30
$(60 \%)$ out of 50 men with PGU had Chlamydiapositive cultures. This differs highly significantly $\left(\chi_{1}^{2} 16.97, P<0.001\right)$ from those men with no PGU. Furthermore $30(96.8 \%)$ out of 31 patients with Chlamydia-positive cultures who were followed up developed PGU, while only $20(48.8 \%)$ of 41 patients with Chlamydia-negative cultures developed PGU. This difference is highly significant $\left(\chi_{1}{ }^{2} 16 \cdot 97\right.$, $P<0.001$ ).

TREATMENT OF GU AND DEVELOPMENT OF PGU Gonococci were eradicated with each of the treatments used. Of the 31 patients treated with procaine penicillin $(2.4$ megaunits, intramuscularly) $20(64.5 \%)$ developed PGU; of these latter 12 $(60 \%)$ were Chlamydia-positive, but only one $(9.1 \%)$ of the 11 patients without PGU was Chlamydia-positive. This difference is almost significant $\left(\chi^{2} 5 \cdot 61, \mathrm{P}<0.05\right)$. Thus 12 out of 13 patients with Chlamydia-positive cultures and treated with procaine penicillin developed PGU (Table).

Of 41 patients treated with gentamicin $(280 \mathrm{mg}$, intramuscularly) or spectinomycin ( $2 \mathrm{~g}$, intramuscularly) $30(73.2 \%)$ developed PGU; of these 18 had Chlamydia-positive cultures. Of the 22 patients with Chlamydia-negative cultures $12(54.5 \%)$ had PGU. Thus PGU developed significantly $\left(\chi^{2} 8.62, P<0.01\right)$ more often in patients with Chlamydia-positive cultures than in those with Chlamydia-negative cultures.

Of the five patients (Figure) who became Chlamydia-positive after treatment for PGU, two were treated with procaine penicillin, one with spectinomycin, and two with gentamicin. In three patients the initially Chlamydia-positive cultures became Chlamydia-negative despite the development of PGU. One of these patients was treated with procaine penicillin and two with spectinomycin. All the cultures from the other 22 patients who also had initially Chlamydia-positive cultures remained so during the follow-up visit.

Table Number of patients with gonococcal urethritis and postgonococcal urethritis in relation to isolation of C. trachomatis and antibiotic treatment

\begin{tabular}{|c|c|c|c|c|c|}
\hline \multirow[b]{2}{*}{ Treatment } & \multicolumn{2}{|c|}{ Chlamydia-positive } & \multicolumn{2}{|c|}{ Chlamydia-negative } & \multirow[b]{2}{*}{ Total } \\
\hline & $P G U$ & No $P G U$ & $P G U$ & No $P G U$ & \\
\hline $\begin{array}{l}\text { Procaino penicillin } \\
(2.4 \text { megaunits, im }\end{array}$ & 12 & 1 & 8 & 10 & 31 \\
\hline $\begin{array}{l}\text { Spectinomycin } \\
(2 \mathrm{~g}, \mathrm{im})\end{array}$ & 11 & $\mathbf{0}$ & 10 & 8 & 29 \\
\hline $\begin{array}{l}\text { Gentamicin } \\
(280 \mathrm{mg}, \mathrm{im})\end{array}$ & 7 & 0 & 2 & 3 & 12 \\
\hline Total & 30 & 1 & 20 & 21 & 72 \\
\hline
\end{tabular}




\section{Discussion}

The role of $C$. trachomatis as the cause of PGU has been studied during recent years. Richmond et al. (1972) found that $32 \%$ of their 99 patients with GU had associated chlamydial infections, and $81 \%$ of those patients with Chlamydia-positive cultures who were followed up developed PGU.

The incidence of $C$. trachomatis in patients with GU was $25 \%$ in the study of Oriel et al. (1976) and $15.5 \%$ in a recent study of Alani et al. (1977). In the present study the isolation rate of $C$. trachomatis was $28.6 \%$ (38 out of 133 patients) in patients with GU. Among those patients who were followed up after the treatment of GU, $50(69.4 \%)$ out of 72 developed PGU. The diagnosis of PGU was made a week after treatment, and possibly even more patients would have developed PGU if the follow-up period had been longer (Vaughan-Jackson et al., 1977).

When studying the isolation rate of a possible urethral pathogen the choice of a control group is important. The isolation rate of $C$. trachomatis in patients with NSU is often compared with that in patients attending a venereal diseases clinic but without urethritis. In our laboratory the isolation rate of C. trachomatis was 93 out of $159(58.5 \%)$ in patients with NSU and 0 out of 69 in similar patients matched for promiscuity (Terho, 1978a).

Vaughan-Jackson et al. (1977) used their patients with GU who did not develop PGU as a control group. In this present study 22 patients out of 72 did not develop PGU, and only one had a Chlamydiapositive culture. This group differed highly significantly from those developing PGU, of whom $60 \%$ (30 out of 50) had Chlamydia-positive cultures $\left(\chi_{1}^{2} 16.97, P<0.001\right)$. These findings support the aetiological role of $C$. trachomatis as a urethral pathogen in PGU.

Since isolation of $C$. trachomatis by the cell culture method has become possible in more laboratories (Reeve et. al., 1975, Terho, 1978b) and since this technique has also been adapted to test antibiotic sensitivity in chlamydial strains (Ridgway et al., 1976, Kuo et al., 1977), it is of interest to compare the development of PGU in patients with Chlamydia with that in patients with GU but without Chlamydia and treated with different antimicrobials. Among the antibiotics most widely used for the treatment of GU penicillins (procaine penicillin or ampicillin) show only marginal efficacy against C. trachomatis (Kuo et al., 1977, Blackman et al., 1977) and amino-glycosides (gentamicin, kanamycin, and spectinomycin) are totally ineffective (Ridgway et al., 1976). Thus Oriel et al. (1975) found that all their 15 patients with Chlamydia- positive GU developed PGU after treatment with gentamicin, while only 11 out of 29 patients without Chlamydia developed PGU. Later Oriel et al. (1977) showed that the treatment of GU with spectinomycin was associated with PGU in all 17 patients with Chlamydia, but again only eight $(17.4 \%)$ out of 46 patients without Chlamydia developed PGU.

Treatment of GU with ampicillin does not eradicate urethral $C$. trachomatis as shown by Oriel et. al. (1976), who found that PGU developed in 12 out of 15 patients with Chlamydia-positive GU treated with ampicillin $(2 \mathrm{~g})$ plus probenecid (1 g). Johannisson et al. (1977), from Sweden, treated their patients with GU with an ampicillin derivate, pivampicillin, and found that 11 (73\% out of 15 patients who developed PGU had Chlamydiapositive cultures. Recently Vaughan-Jackson et al. (1977) confirmed the findings of Oriel et al. by treating GU with kanamycin or procaine penicillin. They also found that three out of 11 patients treated with procaine penicillin and seven out of 19 patients treated with kanamycin had Chlamydia-positive cultures after treatment. As the Table shows almost all (30 out of 31) patients with Chlamydiapositive GU developed PGU regardless of the treatment.

The present study also confirms the previous findings that recommended antibiotic treatment schedules, using procaine penicillin or spectinomycin, are ineffective in eradicating $C$. trachomatis. The results should be taken into consideration when new types of treatment for GU are envisaged, especially when spectinomycin-an antibiotic which is totally ineffective against $C$. trachomatis-is recommended for the treatment of $\beta$-lactamaseproducing gonococci (Vaughan-Jackson et al., 1977).

Clinicians should also follow up patients carefully after any type of treatment for GU and investigate them thoroughly for evidence of PGU, for it is often clinically inapparent but may still cause complications similar to those of NSU (Oriel, 1976).

The author thanks Ms Anni Heikkilä and Ms Ulla Virtanen for help during the collection of specimens and Ms Tuula Lindholm for excellent laboratory assistance.

\section{References}

Alani, M. D., Darougar, S., Burns, D. C. MacD., Thin, R. N., and Dunn, H. (1977). Isolation of Chlamydia trachomatis from the male urethra. British Journal of Venereal Diseases, 53, 88-92.

Blackman, H. J., Yoneda, C., Dawson, C. R., and Schachter, J. (1977). Antibiotic susceptibility of Chlamydia trachomatis. Antimicrobial Agents and Chemotherapy, 12, 673-677.

Johannisson, G., Edman, B., and Lycke, E. (1977). Chlamydia trachomatis infection and venereal disease. Acta Dermato-venereologica, 57, 455-458. 
Johnson, F. W. A., and Hobson, D. (1977). The effect of penicillin on genital strains of Chlamydia trachomatis in tissue culture. Journal of Antimicrobial Chemotherapy, 3, 49-56.

Kuo, C-C., Wang, S-P., and Grayston, J. T. (1977). Antimicrobial activity of several antibiotics and a sulfonamide against Chlamydia trachomatis in cell culture. Antimicrobial Agents and Chemotherapy, $12,80-83$.

Oriel, J. D. (1976). Nature, diagnosis and management of nonspecific urethritis. Bulletin of the New York Academy of Medicine, $52,877-885$.

Oriel, J. D., Reeve, P., Powis, P., Miller, A., and Nicol, C. S. (1972) Chlamydial Infection. Isolation of Chlamydia from patients with non-specific genital infection. British Journal of Venereal Diseases, 48, 429-436.

Oriel, J. D., Reeve, P., Thomas, B. J., and Nicol, C. S. (1975). Infection with Chlamydia Group $A$ in men with urethritis due to Neisseria gonorrhoeae. Journal of Infectious Diseases, 131, 376-382.

Oriel, J. D., Ridgway, G. L., Tchamouroff, S., and Owen, J. (1977). Spectinomycin hydrochloride in the treatment of gonorrhoea: its effect on associated Chlamydia trachomatis infections. British Journal of Venereal Diseases, 53, 226-229.
Reeve, P., Owen, J., and Oriel, J. D. (1975). Laboratory procedure for the isolation of Chlamydia trachomatis from the human genital tract. Journal of Clinical Pathology, 28, 910-914.

Richmond, S. J., Hilton, A. L., and Clarke, S. K. R. (1972). Chlamydial infection. Role of Chlamydia Subgroup $\mathbf{A}$ in non-gonococcal and post-gonococcal urethritis. British Journal of Venereal Diseases, 48, 437-444.

Ridgway, G. L., Owen, J. M., and Oriel, J. D. (1976). A method for testing the antibiotic susceptibility of Chlamydia trachomatis in cell culture system. Journal of Antimicrobial Chemotherapy, 2, 71-76.

Terho, P. (1978a). Chlamydia trachomatis in non-specific urethritis. British Journal of Venereal Diseases, 54, 251-256.

Terho, P. (1978b). Isolation techniques of Chlamydia trachomatis from patients with nonspecific urethritis. Dermatologische Monatschrift, 164, 515-520.

Vaughan-Jackson, J. D., Dunlop, E. M. C., Darougar, S., Treharne, J. D., and Taylor-Robinson, D. (1977). Urethritis due to Chlamydia trachomatis. British Journal of Venereal Diseases, 53, 180-183. 